\title{
Eficiência de deposição de agrotóxicos obtida com bocal eletrostático para pulverizador costal motorizado(1)
}

\author{
Aldemir Chaim ${ }^{(2)}$, Maria Conceição Peres Young Pessoa( ${ }^{(2)}$ e Vera Lúcia Ferracini(2) \\ Resumo - O grande desperdício das atuais técnicas de aplicação de agrotóxicos tem estimulado a \\ procura por alternativas tecnológicas para aumentar sua eficiência e a pulverização eletrostática tem se \\ revelado uma tecnologia promissora. O objetivo deste trabalho foi avaliar um bocal eletrostático adap- \\ tável em pulverizador costal motorizado, e verificar a influência da tensão de indução e vazão de líquido \\ na intensidade da carga das gotas, bem como o efeito da carga na deposição de traçador. O estudo da \\ deposição foi realizado com alvo artificial esférico. A carga máxima obtida com o bocal eletrostático foi \\ de 4,0 $\mathrm{mC} / \mathrm{kg}$ para uma vazão de calda de $0,3 \mathrm{~L} / \mathrm{min}$ e tensão de $8,0 \mathrm{kV}$. O aumento na vazão da calda de \\ pulverização reduziu a carga das gotas. Gotas sem carga apresentaram uma eficiência de deposição de \\ $18 \%$ de traçador, mas as gotas com intensidade de carga de $4,0 \mathrm{mC} / \mathrm{kg}$ aumentaram significativamente a \\ deposição para $62 \%$ do total do traçador aplicado. \\ Termos para indexação: produtos agroquímicos, resíduos, pulverização eletrostática, métodos de apli- \\ cação.
}

Pesticide efficiency deposition obtained with electrostatic spray head for knapsack mistblower sprayer

\begin{abstract}
The great losses of the current pesticide application technique has stimulated a search for technological alternatives to increase its efficiency, and the electrostatic spraying has shown itself a very promising technology. The objective of this work was to evaluate an electrostatic spray head for use with knapsack mistblower sprayer and to verify the influence of the induction tension and liquid flow rate, in the droplet charge intensity, as well as the effect of the charge on the tracer deposition. The study of the deposition was accomplished with spherical artificial targets. The maximum charge obtained with the electrostatic head was of $4.0 \mathrm{mC} / \mathrm{kg}$, for spray flow rate of $0.3 \mathrm{~L} / \mathrm{min}$ and tension of $8.0 \mathrm{kV}$. The increase in the spray flow rate reduced the droplet charge. The droplets without charge presented a tracer deposition efficiency of $18 \%$, but the droplets with charge intensity of $4.0 \mathrm{mC} / \mathrm{kg}$ increased significantly the deposition for $62 \%$ of the total amount of the applied tracer.
\end{abstract}

Index terms: agricultural chemicals, residues, electrostatic spraying, application methods.

\section{Introdução}

Existe um grande interesse na redução dos impactos ambientais proporcionados pela agricultura, bem como uma forte demanda de alternativas que permitam a sua sustentabilidade. O método de aplicação de defensivos mais empregado atualmente é extremamente desperdiçador, não adequado, portanto, ao novo paradigma proposto. Exemplos de des-

\footnotetext{
(1) Aceito para publicação em 18 de julho de 2001

(2) Embrapa-Centro Nacional de Pesquisa de Monitoramento e Avaliação de Impacto Ambiental, Caixa Postal 69 CEP 13820-000 Jaguariúna, SP.

E-mail: aldemir@cnpma.embrapa.br,

young@cnpma.embrapa.br, vera@cnpma.embrapa.br
}

perdícios têm sido constatados em alguns trabalhos científicos para culturas de porte rasteiro. Assim, Pessoa \& Chaim (1999) constataram perda de 50\% na deposição de herbicidas em aplicação aérea em arroz irrigado. Chaim et al. (1999c) observaram que, dependendo do porte das plantas, as perdas de agrotóxicos na cultura do feijão permaneceram entre 49 a $88 \%$, e na cultura do tomate, entre 44 e $71 \%$.

Em culturas de porte arbustivo, como a videira, as perdas de agrotóxicos no solo variaram entre 35 e 49\% (Pergher et al., 1997). Em tomate estaqueado, Chaim et al. (1999a) detectaram que a porcentagem de agrotóxico depositado nas plantas variou entre 24 e $41 \%$. As perdas no solo ficaram entre 20 e $39 \%$, e cerca de 30 a $45 \%$ perdeu-se provavelmente por evaporação ou deriva. 
Hislop (1988), numa revisão sobre pulverização eletrostática na agricultura, constatou que ela permite redução de $50 \%$ no uso dos agrotóxicos, sem diminuir a eficácia biológica nos tratamentos fitossanitários.

Chaim (1998) e Chaim et al. (1999b) desenvolveram bicos pneumáticos eletrostáticos para aplicação de caldas aquosas na produção de pequenas gotas com alta intensidade de carga. Entretanto, os bicos desenvolvidos pelos autores exigiam uma elevada pressão de ar, inviabilizando sua utilização em equipamentos costais.

O objetivo deste trabalho foi avaliar um bocal eletrostático adaptável em pulverizador costal motorizado, e verificar a influência da tensão de indução e vazão de líquido na intensidade de carga das gotas, bem como o efeito da carga na deposição de traçador.

\section{Material e Métodos}

Um protótipo de bocal eletrostático utilizando processo de carga por indução, de acordo com os princípios descritos por Chaim (1998) e Chaim (1999b), foi adaptado a um pulverizador costal motorizado Hatsuta, modelo BM15 (Figura 1).
Uma fonte de alta tensão de corrente contínua, variável entre 0 e $30 \mathrm{kV}$, alimentada por bateria de $9 \mathrm{~V}$, foi utilizada para fornecer o potencial necessário ao eletrodo de indução do bocal. A alta tensão foi monitorada com um Voltímetro ICEL modelo SK 9000.

A intensidade da carga das gotas foi medida segundo Chaim (1998) e Chaim et al. (1999b), com o auxílio de um multímetro digital Minipa ET 2060. Para verificar a influência da tensão na intensidade de carga das gotas, a vazão de líquido do bocal eletrostático foi mantida em $0,3 \mathrm{~L} / \mathrm{min}$. Na verificação da influência da vazão do líquido na intensidade de carga das gotas, a tensão aplicada ao bocal ficou mantida em $8,0 \mathrm{kV}$.

No teste de deposição em alvo artificial, foram usadas esferas de isopor de $0,2 \mathrm{~m}$ de diâmetro, recobertas com folha de alumínio, aterradas ao solo por haste metálica de $0,6 \mathrm{~m}$ de altura. A amostragem da deposição foi realizada com quatro cartões de papel do tipo mata-borrão, com gramatura de $250 \mathrm{~g} / \mathrm{m}^{2}$, medindo $2,0 \times 5,0 \mathrm{~cm}$. Os cartões foram fixados no plano horizontal da região mediana de cada esfera de amostragem, em quatro pontos eqüidistantes, de maneira que ficassem dispostos em quatro lados opostos.

Este ensaio foi realizado com cinco tratamentos correspondentes a intensidades de carga das gotas $(0,0,1,0,2,0$, 3,0 e $4,0 \mathrm{mC} / \mathrm{kg}$ ) e 20 repetições. Em cada tratamento,

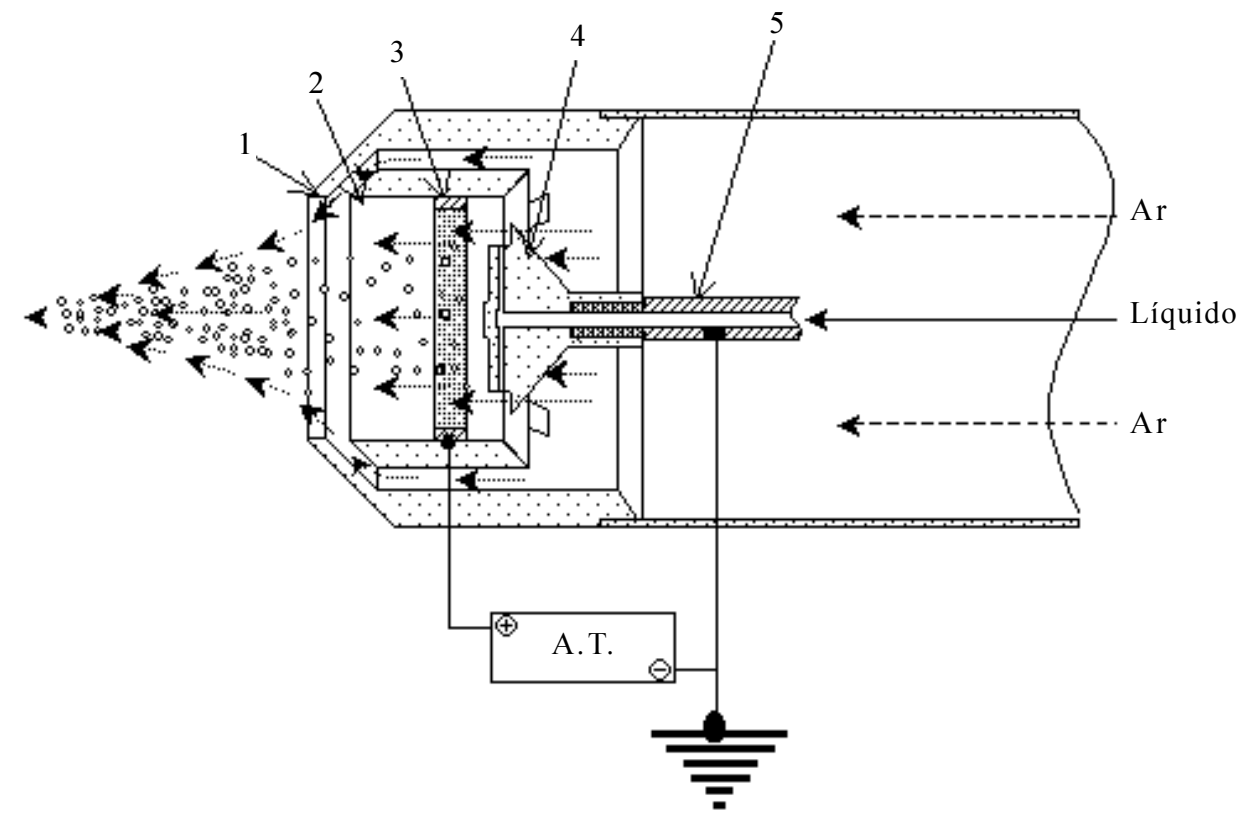

Figura 1. Esquema do bocal pneumático eletrostático, para uso em pulverizadores costais motorizados onde são representados: 1: saída do ar; 2: suporte de sustentação do eletrodo de indução; 3 : eletrodo de indução; 4: "margarida" de pulverização; 5: tubulação de condução do líquido; A.T : fonte de alta tensão. 
foram utilizadas duas fileiras de dez esferas, com espaços, entre si, de 0,5 m de distância, formando uma faixa retangular de 5,0 m de comprimento por $0,5 \mathrm{~m}$ de largura. As esferas foram dispostas de maneira que dois dos alvos de cartão mata-borrão ficassem alinhados com a fileira. As aplicações foram iniciadas a 27,5 m antes do conjunto das esferas de amostragem e concluídas a igual distância, de maneira que o percurso total em cada passagem do aplicador fosse de 60 metros. A pulverização de cada tratamento foi realizada em duas passagens do aplicador, do lado externo de cada fileira de amostragem da faixa retangular do conjunto de esferas, com o bocal mantido a $0,6 \mathrm{~m}$ de distância das laterais. Foi mantido um posicionamento perpendicular do bocal em relação a cada fileira, de maneira que apenas um dos quatro cartões de amostragem de cada esfera recebesse uma deposição direta do fluxo de gotas. A velocidade de deslocamento do aplicador foi regulada para $60 \mathrm{~m} / \mathrm{min}$, e a vazão do equipamento, calibrada para $0,3 \mathrm{~L} / \mathrm{min}$, a fim de possibilitar um consumo estimado de $100 \mathrm{~L}$ de calda por hectare.

Durante as aplicações, a velocidade do vento foi medida com anemômetro manual Davis, modelo Turbo Meter. As condições de temperatura e umidade relativa foram monitoradas com um termoigrômetro manual Sper Scientific 800016.

Como traçador, foi usado um fungicida cúprico, contendo em sua formulação o equivalente a $350 \mathrm{~g}$ de cobre metáli$\mathrm{co} / \mathrm{kg}$. Cinco amostras das caldas preparadas para obtenção de concentração $1.000 \mathrm{mg} / \mathrm{L}$ de cobre metálico foram utilizadas para a confirmação do teor do traçador. Após as aplicações, as análises do traçador nas amostras foram realizadas segundo Chaim et al. (1999a), com a quantidade de cobre determinada por espectrofotômetro Shimadzu AA 380.

\section{Resultados e Discussão}

O teste para avaliar a influência da tensão de indução na intensidade de carga das gotas apresentou curva de resposta semelhante às obtidas por Chaim (1998) e Chaim et al. (1999b). Entretanto, o valor máximo da carga obtida foi de $4,0 \mathrm{mC} / \mathrm{kg}$ para a vazão de $0,3 \mathrm{~L} / \mathrm{min}$ e tensão de $8 \mathrm{kV}$ (Figura 2). A carga das gotas aumentou até atingir um patamar a partir do qual os incrementos, na tensão de indução, foram prejudiciais ao processo de eletrificação. De acordo com as descrições de Chaim (1984), existe um processo de carga por efeito "corona" na qual as partículas adquirem carga da mesma polaridade do eletrodo submetido a alta tensão. Considerando a geometria do bocal eletrostático, o eletrodo de indução e a região de formação das gotas ficaram distanciados por aproximadamente $8,0 \mathrm{~mm}$ (Figura 1). Desta forma as tensões acima de $9 \mathrm{kV}$ resultaram em um intenso campo eletrostático, com conseqüente ionização do ar com cargas da mesma polaridade do eletrodo de indução, que neutralizaram parcialmente a eletrificação das gotas.

Houve uma redução linear na carga das gotas, no incremento de vazão dentro da faixa compreendida entre 0,1 e 0,7 L/min (Figura 3). Essa relação inversa entre a carga e vazão, também observada por Chaim (1984, 1998) e Chaim et al. (1999b), tem sido atribuída ao aumento do diâmetro das gotas. No caso do bocal eletrostático, a velocidade do vento responsável pela pulverização foi constante, e, conseqüentemente, o aumento da vazão do líquido proporcionou a formação de gotas maiores, com cargas menores.

As análises do teor de cobre de cinco amostras da calda utilizada nos testes de deposição demonstraram variações compreendidas entre 999,5 e $1.000,6 \mathrm{mg} / \mathrm{L}$, indicando que as pesagens do fungicida e as diluições foram precisas.

Considerando que o consumo estimado de cobre metálico foi de $100.000 \mathrm{mg} /$ ha e que a somatória das

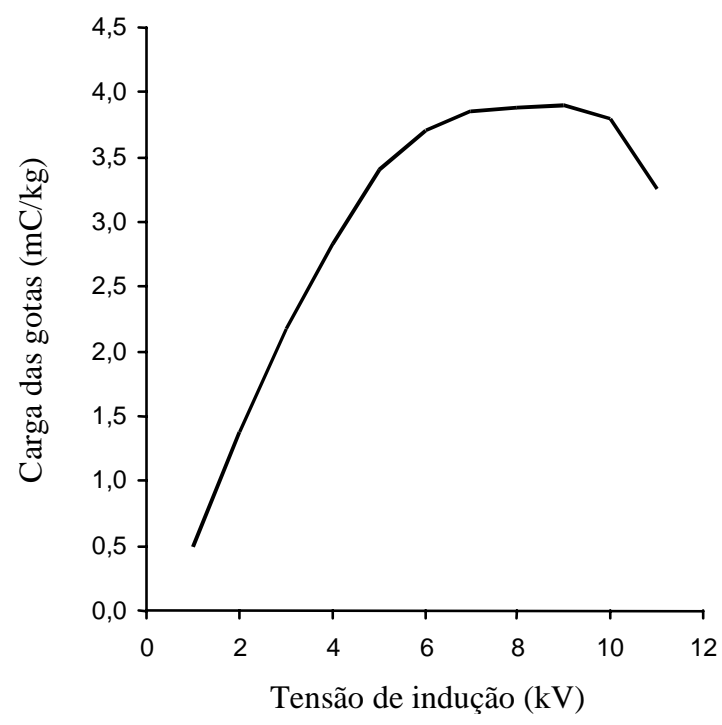

Figura 2. Relação entre intensidade de carga das gotas e tensão de indução, obtida com bocal eletrostático calibrado com vazão de líquido de $0,3 \mathrm{~L} / \mathrm{min}$. 
áreas de superfície das esferas foi de $5.027 \mathrm{~m}^{2} / \mathrm{ha}$, a quantidade teórica de traçador que deveria depositar na superfície dos alvos seria de $19,89 \mathrm{mg} / \mathrm{m}^{2}$. Com a pulverização sem carga, a quantidade média de traçador depositada foi de $3,6 \mathrm{mg} / \mathrm{m}^{2}$, e isso representou uma eficiência de deposição de apenas $18 \%$ em relação à quantidade teórica esperada (Tabela 1). Essa baixa eficiência é semelhante às eficiências obtidas por Chaim et al. (1999 a, 1999c), que constataram perdas de $76 \%$ de agrotóxicos na cultura do tomate estaqueado com $0,5 \mathrm{~m}$ de altura, e $88 \%$ na cultura do feijão, com $0,15 \mathrm{~m}$ de altura. Entretanto, a pulverização eletrostática com intensidade de carga de 4,0 $\mathrm{mC} / \mathrm{kg}$ depositou $12,34 \mathrm{mg} / \mathrm{m}^{2}$, aumentando a eficiência de deposição do traçador para $62 \%$, ou seja, três vezes superior à obtida com as gotas sem carga. Como apenas um dos cartões de amostragem de cada esfera ficou diretamente exposto ao fluxo de gotas durante as pulverizações, os grandes incrementos de deposição só aconteceriam se os outros três cartões de amostragem recebessem alguma quantidade de traçador. Portanto, os resultados demonstram, indiretamente, que as forças eletrostáticas também melhoram a distribuição da deposição dos agrotóxicos nos alvos (Tabela 1). Law \& Cooper (1988) relataram que as deposições das pulverizações eletrostáticas podem ser sete vezes maiores do que as obtidas com as gotas descarregadas, dependendo das características morfológicas do alvo e da própria intensidade de carga das gotas. No caso do bocal eletrostático, que foi testado com alvo totalmente esférico, a força aerodinâmica do fluxo de ar do pulverizador pode ter sido superior à atração eletrostática das gotas reduzindo, a deposição, conforme a teoria apresentada por Law \& Cooper (1988).

As condições micrometeorológicas, monitoradas durante o experimento de deposição, apresentaram variação na velocidade de vento entre 0,0 e $0,1 \mathrm{~m} / \mathrm{s}$, temperatura entre $24 \mathrm{e} 26^{\circ} \mathrm{C}$ e umidade relativa entre 65 e $68 \%$. A influência do vento atmosférico na perda de deposição por deriva foi desprezível, mas as condições de temperatura e umidade relativa podem ter contribuído para evaporação de gotas. A evaporação de gotas foi igual em todos os tratamentos, e não influenciou nas diferenças entre os resultados de deposição.
Os resultados obtidos com o bocal eletrostático comprovam que é possível reduzir os desperdícios de agrotóxicos para o meio ambiente, reduzindo, conseqüentemente, os seus impactos negativos. O aumento na deposição do agrotóxico proporcionado pela pulverização eletrostática pode propiciar uma significativa redução das doses normalmente aplicadas (Hislop, 1988), diminuindo o custo de produção e melhorando a qualidade dos alimentos.

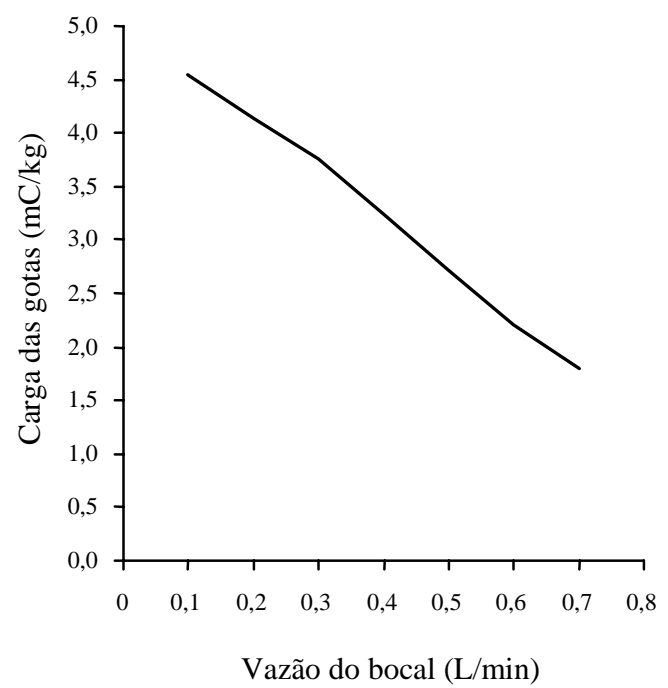

Figura 3. Relação entre intensidade de carga das gotas e vazão do líquido, obtida com bocal eletrostático calibrado com tensão de indução de $8 \mathrm{kV}$.

Tabela 1. Deposição de traçador sobre a superfície de alvo artificial esférico de $0,2 \mathrm{~m}$ de diâmetro, obtida com pulverizador costal motorizado, com bocal eletrostático calibrado para uma vazão de calda de $0,3 \mathrm{~L} / \mathrm{min}$ e diferentes intensidades de carga das gotas.

\begin{tabular}{lccccc}
\hline Deposição do traçador $\left(\mathrm{Cu}^{2+}\right)$ & \multicolumn{5}{c}{ Intensidade de carga das gotas } \\
\cline { 2 - 6 } & 0,0 & 1,0 & 2,0 & 3,0 & 4,0 \\
\hline $\begin{array}{l}\text { Quantidade média depositada } \\
\left(\mathrm{mg} / \mathrm{m}^{2}\right)^{(1)}\end{array}$ & $3,6 \mathrm{a}$ & $5,6 \mathrm{~b}$ & $8,4 \mathrm{c}$ & $10,8 \mathrm{~d}$ & $12,3 \mathrm{e}$ \\
Eficiência da deposição $(\%)^{(2)}$ & 18 & 28 & 42 & 54 & 62
\end{tabular}

${ }^{(1)}$ Médias seguidas de letras desiguais diferem pelo teste de Tukey $(\mathrm{P}<0,01)$; DMS $=0,25 .{ }^{(2)}$ Considerada como $100 \%$, a deposição teórica máxima de traçador de $19,89 \mathrm{mg} / \mathrm{m}^{2}$. 


\section{Conclusões}

1. O aumento da tensão de indução aumenta a intensidade de carga das gotas até determinado limite, a partir do qual os incrementos da tensão são prejudiciais à eletrificação das partículas.

2. O aumento da vazão de pulverização diminui a intensidade da carga das gotas.

3. O aumento da carga das gotas aumenta significativamente a deposição da pulverização, em alvos artificiais esféricos.

\section{Referências}

CHAIM, A. Aperfeiçoamento de bico de pulverização eletrostática para geração de gotas com alto nível de carga. Pesquisa Agropecuária Brasileira, Brasília, v. 33, n. 4, p. 463-470, abr. 1998

CHAIM, A. Desenvolvimento de um pulverizador eletroidrodinâmico: avaliação do seu comportamento na produção de gotas e controle de trips (Enneothrips flavens Moulton, 1951), em amendoim (Arachis hypogaea L.). 1984. 107 f. Dissertação (Mestrado) - Universidade Estadual Paulista Júlio Mesquita Filho, Jaboticabal. 1984.

CHAIM, A.; CASTRO, V. L.; CORRALES, F.; GALVÃO, J. A. H.; CABRAL, O. M. R. Método para monitorar perdas na aplicação de agrotóxicos na cultura do tomate. Pesquisa Agropecuária Brasileira, Brasília, v. 34, n. 5, p. 741-747, maio 1999a.

CHAIM, A.; LARANJEIRO, A. J.; CAPALBO, D. M. F. Bico pneumático eletrostático para aplicação de inseticidas biológicos em floresta de eucalipto. Jaguariúna: Embrapa-CNPMA, 1999b. 33 p. (Boletim de Pesquisa, 3).

CHAIM, A.; VALARINI, P. J.; OLIVEIRA, D. A.; MORSOLETO, R. V.; PIO, L. C. Avaliação de perdas de pulverização em culturas de feijão e tomate. Jaguariúna: Embrapa-CNPMA, 1999c. 29 p. (Boletim de Pesquisa, 2).

HISLOP, F. C. Electrostatic ground-rig spraying: an overview. Weed Technology, Lawrence, v. 2, p. 94-104, 1988.

LAW, S. E.; COOPER, S. C. Depositional characteristics of charged and uncharged droplets applied by an orchard air carrier sprayer. Transactions of the ASAE, St. Joseph, v. 31, n. 4, p. 984-989, 1988.

PERGHER, G.; GUBIANI, R.; TONETTO, G. Foliar deposition and pesticide losses from three air-assisted sprayers in a hedgerow vineyard. Crop Protection, Oxford, v. 16, n. 1, p. $25-33,1997$.

PESSOA, M. C. P. Y.; CHAIM, A. Programa computacional para estimativa de uniformidade de gotas de herbicidas aplicados por pulverização aérea. Pesquisa Agropecuária Brasileira, Brasília, v. 34, n. 1, p. 45-56, jan. 1999. 